\title{
A Mental Models Perspective on Designing Information Visualizations for Political Communication
}

\section{Günther Schreder, Florian Windhager, Michael Smuc, Eva Mayr}

Department for Knowledge and Communication Management, Danube University Krems, Austria, guenther.schreder@donau-uni.ac.at

Abstract: Information visualizations (InfoVis) in the context of political communication are designed to convey a broad understanding of socio-political data and their multitude of intricately connected variables to the public. A cognitive framework to explain and empirically study how users acquire and organize their internal representations gained from InfoVis systems with multiple perspectives has not been established. In this paper, we discuss the theory of mental models and its consequences for the design and research of InfoVis interfaces. Especially for multidimensional data, it is a challenge to design accessible and conceptually consistent InfoVis interfaces to support the local and global coherence of the recipients' mental models. In this paper, we exemplarily show how specific design features, i.e., advance organizers, narrative visualizations, seamless transitions, and multiple coordinated views can accomplish this in the field of political communication and its complex data.

Keywords: information visualization, political communication, multidimensional data, mental models, cognition support, sense making

Acknowledgement: This research has been supported by the Austrian Science Fund (FWF), Project No. P28363, and the Austrian Research Promotion Agency (FFG), Project Nos. 835937 and 850751.

\section{Introduction}

Governmental institutions, decision-makers, and media increasingly communicate information and data by providing explorable visualizations to the public. To this end, they employ a large array of applications and interfaces designed to be both easily accessible and attractive to the users they want to reach and convince to take an interest in specific policy-related topics. Socio-political issues frequently relate to a large number of interconnected data that result from the coexistence of different views on controversial topics, diverging interests of stakeholders and extensive research in economics and the social sciences. While media and institutions are expected to objectively relay 
information and to present the points that are most relevant for decisions and laws, this task is becoming increasingly difficult when dealing with complex data. From the point of view of those who want to communicate information, it is a challenge to gain an overview on these complex data and present the resulting political decisions adequately. From the citizen's point of view it can be difficult to relate to political decisions, and to understand the underlying mounds of data. While public political discourse is often characterized by marketing, hidden agendas and principles of persuasion, these mechanisms tend to simplify complex topics and to create highly simplified and biased perspectives. Going far beyond the repertoire of basic statistical charts, websites like The New York Times, The Guardian, or Die Zeit - just to name a few - are offering new ways of visual access to the changing constellations of local or global politics for a public at large, and let users or moderators interactively explore the details of political debates and developments. These interactive graphics can provide insights into political data, systems and processes. As such, information visualization plays an important role in the field of political communication, civic education and journalism (Windhager \& Smuc, 2014).

Information visualization (InfoVis) is defined as "the use of computer-supported, interactive, visual representations of abstract data to amplify cognition" (Card, Mackinlay, \& Shneiderman, 1999, p. 8). InfoVis tools and methods "help us speed our understanding and action in a world of increasing information volumes" (Card, 2008, p. 542). InfoVis tools offer the opportunity to create a deeper understanding of multidimensional data for recipients who might not have detailed domain knowledge or statistical skills. As such, they can serve as a useful tool for citizens and political institutions alike.

Until recently, government data collections have been used as non-public, protected knowledge repositories, to exclusively provide operative knowledge for administrative agencies. In the context of open government data (OGD) initiatives, non-sensitive parts have been opened to the public (Ubaldi, 2013) to raise an administration's transparency and to allow other interested parties access to this vast amount of collected data.

Nevertheless, visualizations of multidimensional data pose their own specific challenges: Looking at the state of research, a widely-accepted theory on how users generate new knowledge from their interaction with information visualizations is still missing (Pohl, Smuc, \& Mayr, 2012). Varying knowledge of users on how to read diagrams or how to interact with tools and interfaces (also generically referred to as "Visual Literacy"), and varying degrees of comprehensibility or usability of images and tools must be considered to achieve a barrier-free communication flow (Windhager \& Smuc, 2014). This is especially challenging in the field of political communication, where the target group of InfoVis tools are not only experts, who manipulate multidimensional data on a daily basis, but the highly heterogeneous group of citizens with different degrees of visual literacy and varying intentions, expectations and prior knowledge. To move towards a more comprehensible and self-explaining design of InfoVis interfaces for public use it is worth to thoroughly investigate how users build up internal representations that are used to gain insights (Smuc et al., 2009), and how the underlying processes of perception, interaction and sensemaking can be supported by cognitively grounded interface design methods. We think that information visualizations in the field of political communication for the public should be designed to convey a 
broad understanding of socio-political data and their multitude of intricately connected variables. This means going beyond the aim to produce information visualizations systems that enable users to read and identify single data points or specific pieces of information in the most efficient way. It also means that instead of addressing experts in narrow, specialized fields, visualizations must be designed for a heterogeneous audience who does not necessarily use them to answer specific questions but want to be informed on a more general level. For this ambition a new theoretical framework as well as suitable empirical methods are needed.

In this paper, we take first steps in this direction by illustrating how successful comprehension processes can be interpreted as the continuous construction of coherent mental models (section 2) and discuss the theory of mental models in the context of InfoVis (section 3). We exemplarily show how contemporary design features can be used to support the coherent construction of mental models in the field of political communication and its complex data (section 4) and present usability methods for the assessment of users' mental models (section 5). Finally, we discuss some implications for the field of political communication (section 6).

\section{Internal Representations between Cognitive Collages and Coherent Mental Models}

In cognitive terms, observations and explorations in geospatial as well as abstract environments can be understood as "the creation and interpretation of an internal mental model" (Spence, 1999, p. 921). Browsing an information space, users continuously build up an internal representation of this space and use this mental model to interpret the information and to explore it in a meaningful way. Mental models are not fixed, but are dynamic representations that enable to "describe, explain and predict a system's purpose, form, function, and state" (Jones, Ross, Lynam, Perez, \& Leitch, 2011, p. 46). The structure of mental models can develop over time, growing richer in content and level of abstraction, allowing them to be more easily transferred to new content or environments. Therefore, mental models are used to describe and explain domain-specific learning or comprehension processes.

Yet it can be assumed that comprehension processes do not necessarily result in the creation of complete and coherent mental models. Tversky (1993) coined the term cognitive collages as a metaphor for the distorted mix-up of partial information, differing perspectives and reference points that characterize fragmentary internal representations. Snippets of information are stored in memory, but are not systematically or only loosely related to one another. Though this information can be recalled, it is difficult to use such ill-structured information to solve more complex problems. In contrast to cognitive collages, mental models integrate different aspects and perspectives and "capture the categorical spatial relations among elements coherently, allowing perspective-taking, reorientation, and spatial inferences" (Tversky, 1993, p. 15). Cognitive science research points to significant payoffs, deliberate use of coherence techniques can have on visual sensemaking: Users with more coherent mental models recall information better (McNamara, et al., 1996) and can better navigate the information space (Tversky, 2011). This allows them also to generate more inferences and gain novel insights into the data. But when do we build up better 
connected and consistent mental models and when do our internal representations remain fragmentary cognitive collages? The continuous and coherent construction seems to be a critical factor for effective mental representations and needs to be supported and facilitated in different modes of communication. To reach this goal, we transfer considerations on mental models and coherence in text comprehension to the design of InfoVis systems.

\subsection{Supporting Coherence}

Since the 1980s research on mental models demonstrated that the success of comprehension processes depends on the incremental construction and extension of coherent mental models. In a ground-breaking experiment by Johnson-Laird (1980), participants had to read one of the two texts shown in Table 1 and then draw the corresponding layout. In the continuous order condition (A) subjects built up a mental model (MM) step by step, whereas in the discontinuous order condition (B) subjects could either build two separate mental models and then combine them or store the information in propositional, fragmentary form. According to Johnson-Laird, both possibilities lead to increased cognitive effort in comparison to condition A. As elaborating an existing mental model or building up a mental model step by step needs lower cognitive effort than combining multiple existing ones, the error rate when information is retrieved decreases, and it can be assumed that understanding of spatial configurations is more successful.

Table 1: Experimental Conditions in Johnson-Laird (1980)

\begin{tabular}{|c|c|}
\hline condition A & condition B \\
\hline The knife is in front of the spoon & The glass is behind the dish \\
The spoon is on the left of the glass & The knife is in front of the spoon \\
The glass is behind the dish & The spoon is on the left of the glass \\
\hline
\end{tabular}

How different pieces of information can be connected during the comprehension processes was also the vital question in research on comprehension of discourses and narratives. Coherence was identified as a major factor in comprehension processes (McNamara, Kintsch, Songer, \& Kintsch, 1996). Coherence can be encountered on a local level, i.e., how extracted propositions are coherently related (microstructure), and on a global level of meaning and deeper understanding (macrostructure) which is conceptualized as the construction of so called situation models. Situation models (Dijk \& Kintsch, 1983; Zwaan \& Radvansky, 1998) are mental representations of particular constellations within a story world, resembling narrative scenes or tableaus. Within these representations, the multidimensional spatial as well as abstract information (i.e., the events of the narrative) is sequentially interwoven. The event indexing model (Zwaan, 1999) postulates that newly processed events belonging to a narrative are analyzed according to five processing cues or indices of coherence: time, place, protagonist, cause and goal. They are matched to recently activated situation models; their interconnection leads to an integrated global mental model of the narrative. A higher number of coherence indices eases the continuous updating of the representation and thus the comprehension process, i.e., the construction of a mental model. 
As opposed to such a linear narrative structure, interactive media already pose a challenge for this continuous construction and integration process: The recipient is not led through the information, but navigates a multitude of decision points in his or her own way and processes the pieces of information in no specific order. While narratives can be skillfully constructed to provide sufficient coherence indices, hypermedia does not offer a comparable guidance (Urakami \& Krems, 2012). If subsequently accessed decision points are conceptually unrelated, resulting coherence gaps will interfere with the successful construction of a coherent mental model. It is assumed that these gaps must be filled with inferences based on the recipient's knowledge. Therefore, the use of pre-information in the form of graphical overviews has been proposed (Bezdan, Kester, \& Kirschner, 2013): Similar to an advance organizer (Ausubel, 1960, cf. section 4.1), recipients of a sketch of the structure and content of the available information, construct a rough mental model in the beginning, which is then continuously extended and elaborated based on the actual information

In summary, the construction of a coherent mental model is an incremental process that can be supported by specific additional cues: Pieces of information can be linked by spatial, temporal or semantic relations. In contrast, a lack of connecting cues or coherence indices rather leads to fragmentary representations - so-called cognitive collages (Tversky, 1993) or "a quagmire of charming, but irrelevant pieces of information", as van Merriënboer and Kirschner (2012, p. 161) put it. In the following section, we transfer these considerations from the domain of text comprehension to the domain of InfoVis design and propose that equivalent coherence indices should be considered for InfoVis systems dealing with complex, abstract data.

\section{Construction of Mental Models during Interaction with InfoVis Tools}

Graphic displays are used to convey information on physical objects, but also on abstract concepts and constellations. Visualizations use spatial relations in diagrammatic space congruently to the conceptual relations of abstract entities (e.g., election results, political systems, or international relations), thereby serving as models of actual or theoretical worlds and drawing on users' highlypracticed skills of spatial reasoning to promote comprehension and reasoning related to nonspatial or conceptual entities (Tversky, 2011).

Liu and Stasko (2010) hypothesize that InfoVis methods and images do not only provide an input or scaffold for human reasoning: According to the theories of distributed cognition and model based reasoning, using an InfoVis system reduces the cognitive effort of constructing and manipulating a mental model. A mental model can be complemented by an interactive visualization that acts as an augmentation for cognitive processes. Hegarty (2011) summarized various aspects, how information visualizations support the construction of mental models: (1) Serving as an external storage of information, visualizations reduce the need to remember all information in full detail and thereby free up working memory for other cognitive processes. (2) Visualizations help to organize information, e.g., the representations of entities related in the real world are close in the display, which facilitates search and information integration and serves as coherence cues to be utilized by the gestalt laws of perception. (3) Cognitive processes can be offloaded on perceptual processes; important features of the data are detected by visual pattern 
recognition processes instead of mental manipulations of information and complex computations. (4) Similarly, cognitive processes can be offloaded to interactions with the display, e.g., rotating a visualized object instead of performing mental rotations reduces cognitive load.

Yet to unfold these cognition-supporting functionalities, diagrams and InfoVis systems should be also accessible, consistent and comprehensible on a micro and macro level of visual complexity. Accordingly, the construction of mental models can be supported on the levels of local and global understanding and coherence. In the following, the term local understanding will cover the understanding of single diagrams or views, which allow to build mental models only of simple data - or only of partial aspects of bigger data sets. On the other hand, global understanding refers to the visual and conceptual integration of multiple views, perspectives or diagrams.

\subsection{Fostering Local Understanding: Single Views}

Existing spatialization principles and InfoVis methods (i.e., different types of diagrams from statistical charts to treemaps, networks, etc.) introduce local coherence to data representations: By assigning visual marks (e.g., dots, lines, shapes) to spatial positions, a visual meta-shape is created which can be explored in detail. But it can also be processed integratedly by gestalt perception and pattern recognition processes, which automatically integrate proximal, connected, or similar marks into higher entities. Additional data dimensions can be encoded using different colors, sizes, orientations, etc. of these marks (cf. Fig. 1., center). Designers can foster a diagram's accessibility and ensure its local coherence and consistency by following explicitly stated design rules or codes of conduct, which are controlled by expert communities of InfoVis practice, or by relying on design principles already built into software and data templates (Card, Mackinlay, \& Shneiderman, 1999).

\subsection{Fostering Global Understanding: Multiple Views}

For more complex and multidimensional data sets, multiple perspectives are possible, and each diagrammatic perspective offers only one possible view on the complete data. Yet, to merge them into a coherent bigger picture - and not only into cognitive collages - these isolated images have to be combined into a consistent mental model by the user. From the mental model perspective and a distributed cognition point of view (Liu \& Stasko, 2010), this challenge already has to be tackled at the IV interface level to ensure that the construction of effective internal representations is well supported and facilitated by external means (i.e., well-integrated multiple views).

\section{Supporting the Construction of Complex Mental Models}

In the following we present methods and techniques to support the initial and incremental construction of mental representations across multiple views. We consider these methods to be specifically relevant to foster global sensemaking from abstract, multidimensional data in political communication. Overall, we delineate three different strategies to support local and global understanding: 
First, methods for initial construction of mental models like advance organizers (sections 4.1) illustrate the conceptual relations between multiple views or data dimensions and thus support the initial setup of a locally and globally consistent mental model.

Second, methods for sequential integration, like narrative visualization techniques (sections 4.2) or seamless transitions (sections 4.3) support the incremental elaboration or extension of an already existing mental model.

Third, methods for parallel integration, like coordinated multiple views and coordinated interaction methods (sections 4.4) integrate local views in parallel, and thus provide synchronous visual cues for coherence and integrated understanding.

\subsection{Advance Organizers}

Ausubel (1960) suggested the use of advance organizers as effective means to improve the learning and retention of verbal material. An advance organizer is a pre-sketch of the learning material and allows to integrate all following information into this structure. Within the framework of a theory of mental models, this pre-sketch activates the recipient's prior knowledge which can be used to build a tentative mental model and to further manipulate and elaborate it in working memory. By this means, new information can be connected to existing knowledge; it can be more easily processed and it is more closely tied into existing mental models.

Advance organizers especially support mastery of content in non-linear, unstructured environments like hypermedia (McManus, 2000) or multidimensional information environments (Windhager \& Mayr, 2012): They provide a conceptual overview and facilitate navigation in more complex information spaces, but also raise curiosity and interest. An advance organizer can be graphical or textual, but a graphical representation of this conceptual pre-sketch is claimed to be less ambiguous and more concise than a textual one (Coffey, Hoffman, \& Cañas, 2006).

Advance organizers for information visualizations can be realized in three different ways: (1) They can provide an overview of the data by means of a selected single view (e.g., a small map), or (2) they can present structural information on the data set (i.e., the data dimensions), or (3) they can walk the user through the information visualization system using an exemplary data set. The first option - an overview of the data following a specific Infovis method - is inherent to most information visualizations due to the prevalence of Shneiderman's seminal visual information seeking mantra "overview first, zoom and filter, then details-on-demand" (1996, p. 337). However, this approach is not sufficient for an overview of more complex data sets, which encompass more data dimensions than can be displayed in one information visualization. Here the second function of advance organizers can play a decisive role - providing structural knowledge: InfoVis interfaces which include an advance organizer on the different data dimensions (Fig. 1, left) can support the initial construction and comprehension of individual representations (Fig. 1, center) and help to clarify the connections between them, thus facilitating their integration into global mental models later (Fig. 1, right). The third type of advance organizers - providing guidance via examples (cf. Gurlitt, Dummel, Schuster, \& Nückles, 2012) -, walks the user through the information visualization system based on an exemplary subset of the data. The recipient constructs a 
prototypical mental model step-by-step and can use it later for the elaboration of the whole data set. Thereby, examples are directly related to the next form of mental model support, which we are going to discuss, narratives.

Figure 1: Tracing the incremental transformation of multiple data dimensions from an advance organizer (left), into standard forms of diagrams or single views (center), to be integrated into more complex mental models sequentially (top right) or synchronously as multiple coordinated views (bottom right).

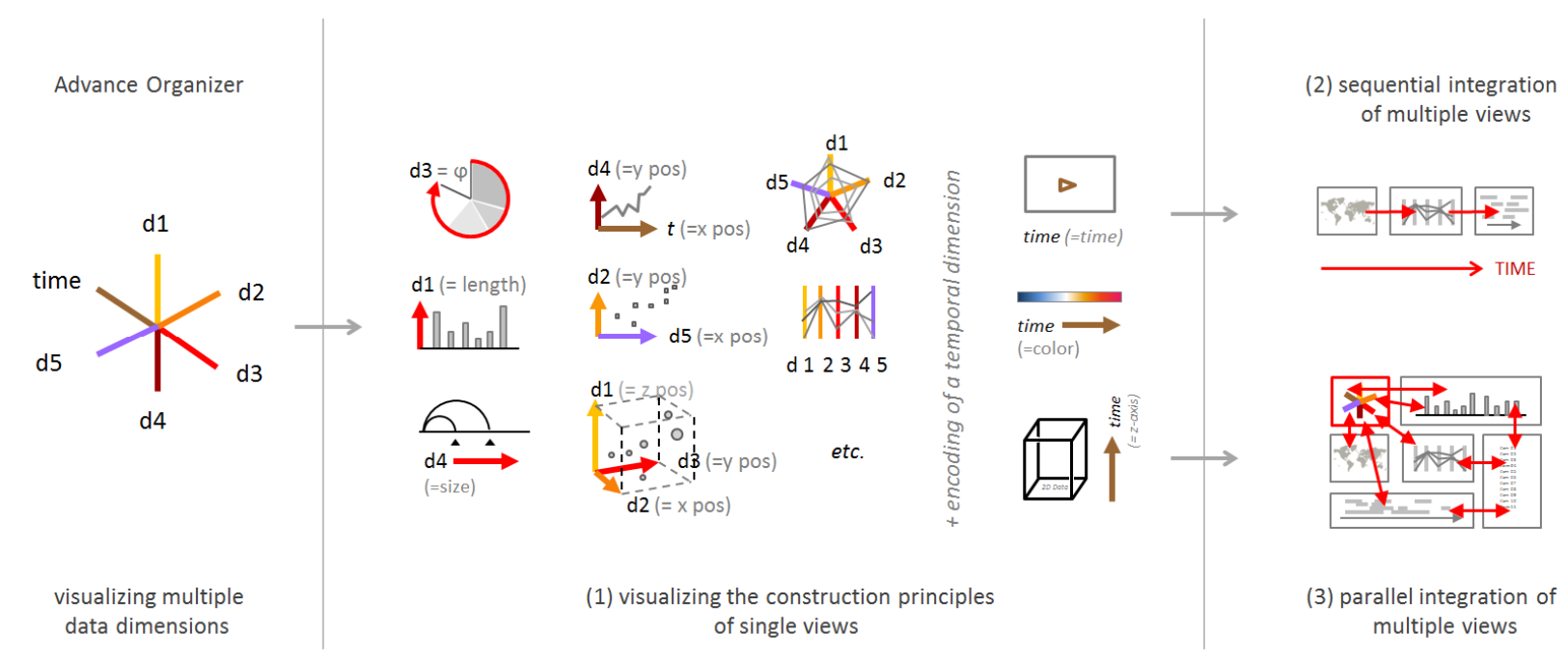

\subsection{Incremental Elaboration of Existing Mental Models through Narrative User Guidance}

When it comes to the elaboration of more complex models, multiple parts or local views have to be connected into a global representation, and one option is to do so sequentially, i.e., by establishing chronological connections between data points. In this context Kosara and Mackinlay (2013) propose moving beyond visual standard techniques for exploration of data towards more effective ways of communicating and presenting data by using elements of storytelling: "Stories have proven to be not only an incredibly popular way of conserving information and passing it on, they also provide the connective tissue between facts to make them memorable" (Kosara \& Mackinlay, 2013 , p. 2). In the context of information visualization, they define a story as a causally related chain of individual visualizations. This ordered sequence often, but not necessarily, corresponds to a chronological course of events and provides the user with a clearly defined path through the data. The authors compare the creation of narrative visualizations with the workflow of journalists - they collect pieces of information and then sequentially tie together the individual parts into a coherent story - and mention the possible beneficial effects of storytelling in visualizations, like engaging the audience or making the visualizations easier to remember.

Based on an analysis of 58 examples, Segel and Heer (2010) analyze the design space of narrative visualizations and identify seven genres (Fig. 2) that vary in number and ordering of 
distinct visual elements, i.e., frames, pictures or text. The genres are not always clearly separated, but can be combined into more complex visualizations, for example by using annotated charts within a slide show format.

Figure 2: Seven Genres of Narrative Visualizations According to Segel and Heer (2010, p. 1145)

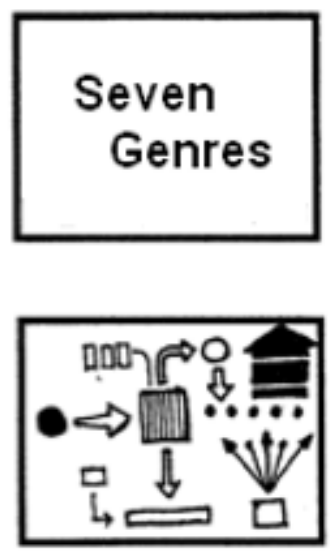

Flow Chart

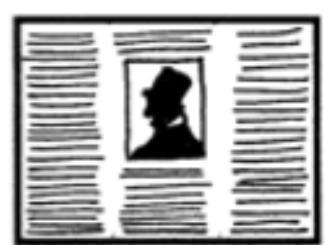

Magazine Style

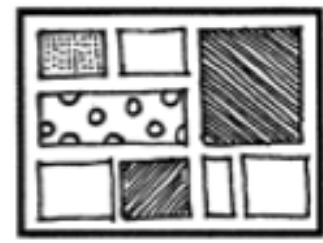

Comic Strip

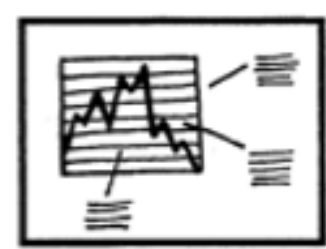

Annotated Chart

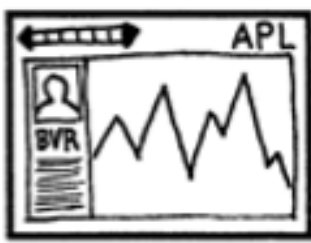

Slide Show

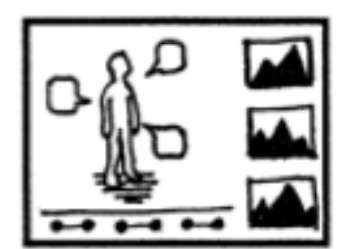

Partitioned Poster

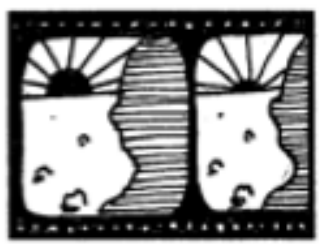

Film/Nideo/Animation

One of the major challenges for the design of any narrative visualization is the balancing of author-driven and reader-driven approaches. Whereas exploration-driven information visualization concepts do not prescribe any specific order or linearity but allow free interaction with the data, an author-driven visualization leads through the material in a predefined way (e.g., as in the cinematic visualization The Fallen of WWII, Halloran, 2015, http://www.fallen.io/ww2/). While most visualizations are either predominantly reader- or author-driven, Segel and Heer (2010) explicate that up-to-date narrative visualizations are characterized by a balance of both approaches. They identify three common design methods that provide the possibility for interactivity embedded in a narrative structure: (a) The "martini glass structure" starts solely author-driven, but opens up to free exploration after the user has been led through the narrative. (b) The "interactive slideshow" walks the user through a linearly arranged slide-show, but individual slides can be explored by the user. (c) The "drill-down story" visualizes a general theme and allows the recipient to choose among different subtopics to explore specific details or backstories which are heavily authored.

Though narrative design became a relevant topic in information visualization, research on the characteristics of the links between the individual elements of a visual narration is still scarce. Studies on comprehension processes in other narrative media provided valuable insights into the nature of the "connective tissue" of narrative visualizations: In their study on film comprehension, Magliano, Miller and Zwaan (2001) found evidence for a general mechanism of constructing situation models based on the event indexing model described in 2.1. In their analysis, the researchers differentiate between temporal continuity, changes of spatial setting, and movement of characters as the main dimensions that provide viewers with informational cues to interpret a sequence of shots as either a contiguous situation or as disconnected events. While the firstdimension correlates to Kosara's and Mackinlay's (2013) description of stories in visualization as a chronologically ordered sequence of events, the latter dimensions highlight the role of spatial 
changes which link the individual elements of a story (cf. section 4.3). For example, in Bloomberg's What's Really Warming the World (Fig. 3) all the line graphs depicting potential causes for global warming encountered during the story are drawn on a persistent spatial layout that retains the line that represents the observed global temperature and allows the user to link the different pieces of information in one coherent mental model.

Figure 3: Roston, E. E Migliozzi, B. (2015). What's Really Warming the World by Bloomberg. URL: http://www.bloomberg.com/graphics/2015-whats-warming-the-world/

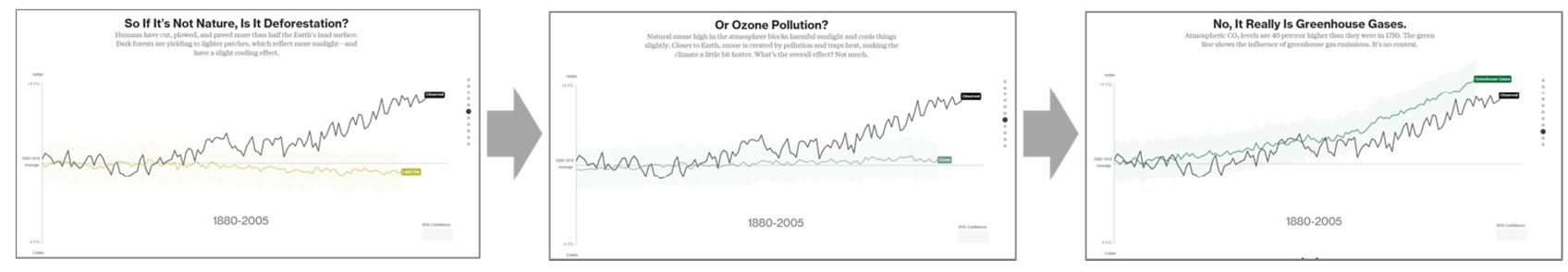

On an abstract level, stories guide recipients through a bigger picture by providing various sequential links between events or places, or generally speaking: between different local data elements. We argue that such narrative walkthroughs can be considered an additional coherence technique for visualizations as the construction of a situation model supports the integration of insights into a coherent mental macro model. Furthermore, we contend that the danger of coherence gaps resulting from the use of interactive media (as explained by Urakami \& Krems, 2012) could be circumvented by the design possibilities for mixed author-driven and reader-driven approaches documented by Segel and Heer (2010), as well as by further coherence techniques described in the following.

\subsection{Incremental Elaboration of Existing Mental Models through Traceable Changes}

As another temporal-sequential coherence technique, seamless transitions produce coherence in terms of visually traceable change (i.e., animation), when switching between spatially or temporally diverse views ${ }^{1}$. This method uses various forms of morphing to visually translate from one spatialization principle to another. By changing layouts incrementally as opposed to cutting abruptly from one view to the next, the spatial re-arrangement can be perceptually traced, and the shifting of relevant, well-known elements into new constellations can be followed smoothly (Fig. 4).

1 According to the seven genre-typology of Segel and Heer (2010, cf. Fig. 2), this technique could also be subsumed to methods of narrative user guidance, but it is addressed here in more detail because of its relevance to bridge between different styles of data encoding in a sequence of multiple views, which are directed on the same set of multidimensional data. 
Figure 4: Smooth transitions leading from a well-known map of a territory (top left) to and abstracted cartogram showing number of eligible voters per federal state (top right), to be transformed into a bar chart showing the voter participation of each state in the elections of Austria of 2012 (Flooh Perlot, 2016, http://www.drawingdata.net/).

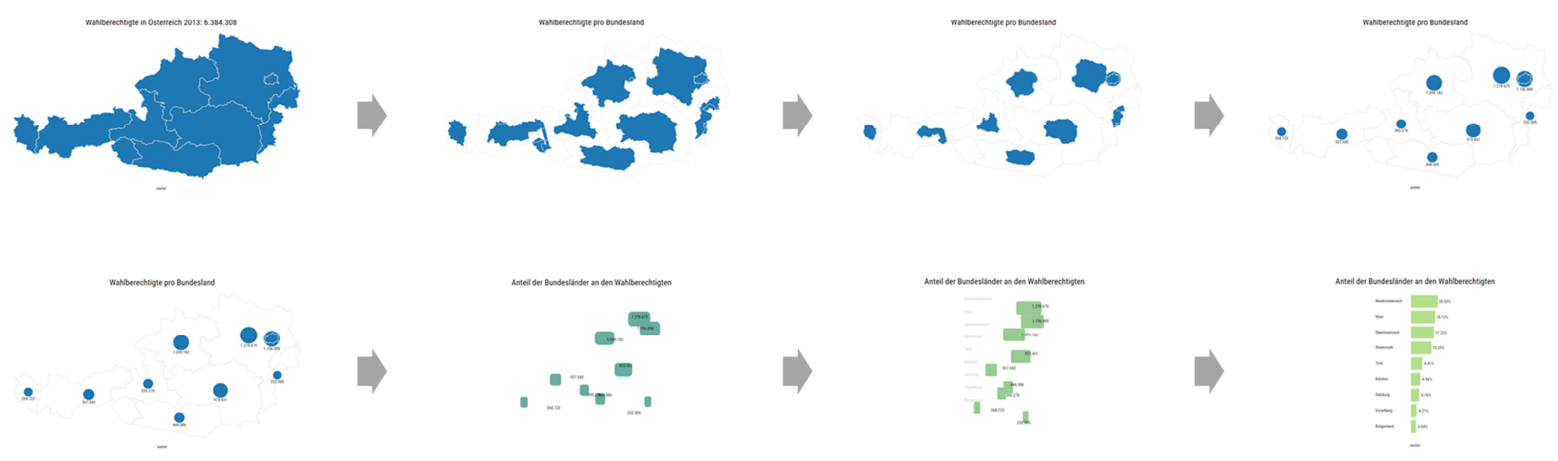

From a cognitive science perspective, these techniques correspond to the concept of preserving the mental map which aims at developing algorithms that keep the number of changing elements to a suitable minimum. Archambault \& Purchase (2013) note that these techniques are especially useful when dealing with complex visualizations with more than around five moving elements. The results from Johnson-Laird's (1980) experiment showed, that it is cognitively more efficient to elaborate one existing mental model, than to structurally recombine two different models. While two separate visualizations can easily lead to two separate mental models, seamless transitions allow the adaptation and extension of an existing MM, resulting in less cognitive effort. Studies on how to support the learning of visualizations by analogy (Ruchikachorn \& Mueller, 2015) provide support for this cognitive-scientific insight from an InfoVis point of view. Windhager and Smuc (2014) outline further possible ways to connect various types of diagrams through seamless transitions in the field of political information visualization (Fig. 5).

\subsection{Parallel Integration of Mental Models (components) with Multiple Juxtaposed Views and Coordinated Interaction Methods}

When multiple (local) views have been successfully constructed, they could be arranged in parallel (i.e., juxtaposed) and linked by coordinated interaction techniques. By offering multiple views in parallel, complex topics or datasets could be presented from complementary analytical perspectives for synchronous contemplation. In contrast to narrative techniques the "connecting tissue" between these views has to be presented in parallel to the observer's eye. One basic technique to do so is the use of consistent visual variables or design choices across different views (e.g., the consistent use of color for the same data dimensions in different perspectives, cf. Fig. 6). Interaction methods subsequently allow the user-driven enrichment of between-view- connections with further coherence cues. The most common of these synchronous integration techniques are coordinated selecting and highlighting or linking and brushing, as well as synchronized panning, scrolling or zooming (North \& Shneiderman, 2000). 
Figure 5: Supporting the preservation of the mental map by smooth transitions between different types of standard diagrams, used in visual communication of political data (cf. Windhager $\mathcal{E}$ Smuc, 2014)

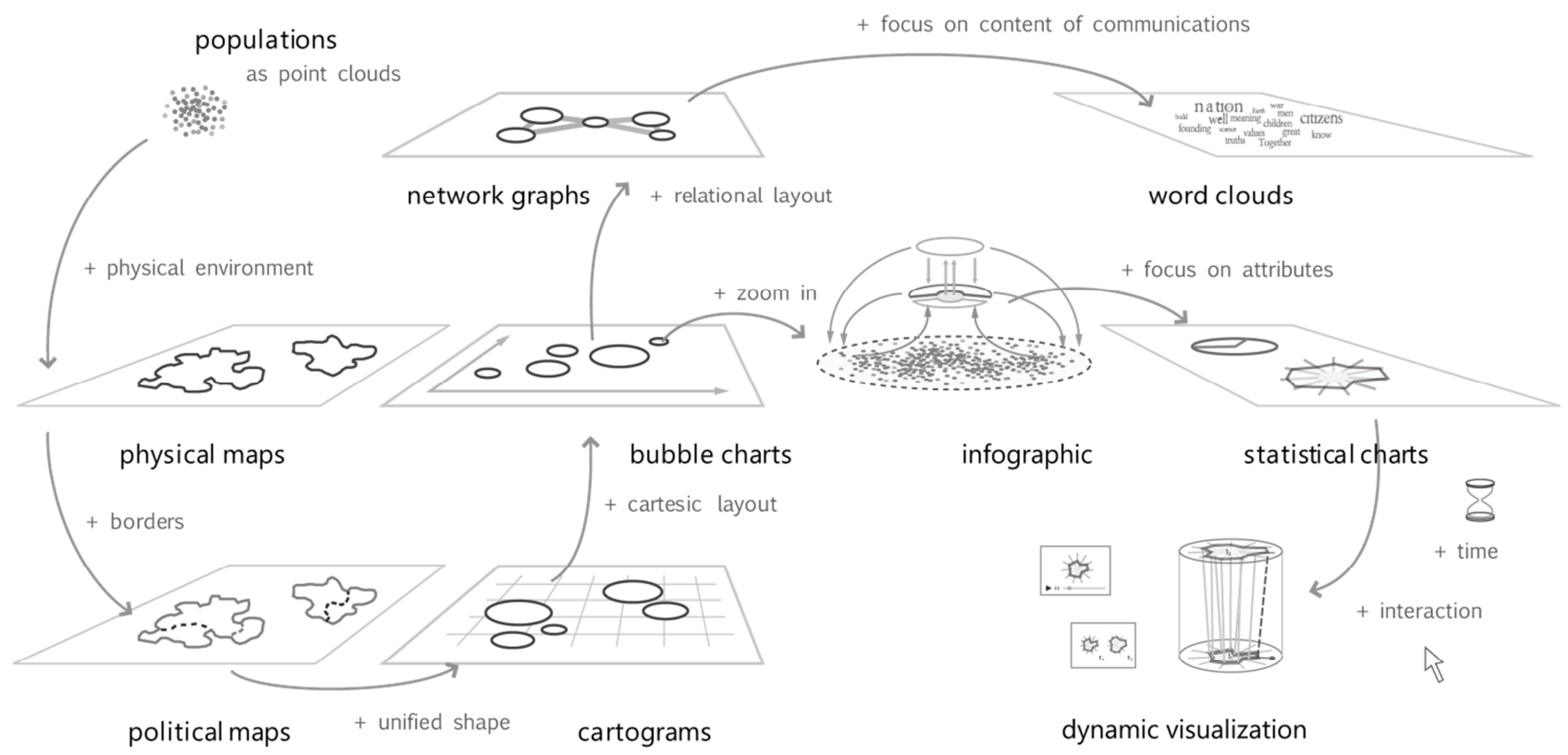

Interaction methods provide instant visual modifications of the same data element in different views, which enable a perceptual integration of different views on demand. Such spatially unconnected but temporally synchronous changes allow relating and comparing selected visual marks or elements in different views, i.e., in different layouts or spatialization contexts or within different temporal selections.

For example, in a project dedicated to the visual analysis of donations in the U.S. election cycle of 2012, multiple visualization methods shed light on various aspects of the same phenomenon (Fig.6). While a map shows the geographic origin of donors (top left), a linked timeline shows the temporal sequence of donations (bottom left). On the right-hand side, two different types of bar charts and a ring chart show the further distribution of donations in general (top right), as well as per congressional chamber (top center), and per campaigning politicians (bottom right). Each of these views could be used to select subsets of the displayed data for closer investigation - and have the same selection displayed in all the other linked views with their own specific focus.

In summary, the design method of multiple coordinated views allows InfoVis designers to present multiple local views or components in parallel, to be integrated by the users' perception in a non-temporal fashion. Even if the perceptual exploration obviously also consumes time, the sequence of the visual scan-path (and therefore on a higher level also of the sensemaking procedure) is at first not determined by such interfaces, until more "author-driven" design elements are added (cf. Segel \& Heer, 2010). As indicated at the end of section 4.2, it is not only possible to combine parallel ("exploration-driven") and sequential ("author-driven") arrangements of multiple local views, but we consider the usable and effective design of such "multiplyintegrated" interfaces as a new high-level design challenge, which deserves specific conceptual 
and empirical attention of its own. As such, these considerations on how to support macrocognition and mental model construction by a more coherent interface design directly lead to the questions, of how such macro-level design choices can be evaluated.

Figure 6: The MapD interface, facilitating a closer look at donations to campaigning politicians in the election cycle of 2012, utilizing a point map, a timeline, as well as bar and ring charts for the parallel view on the same phenomenon (http://www.mapd.com/demos/political-donations/).

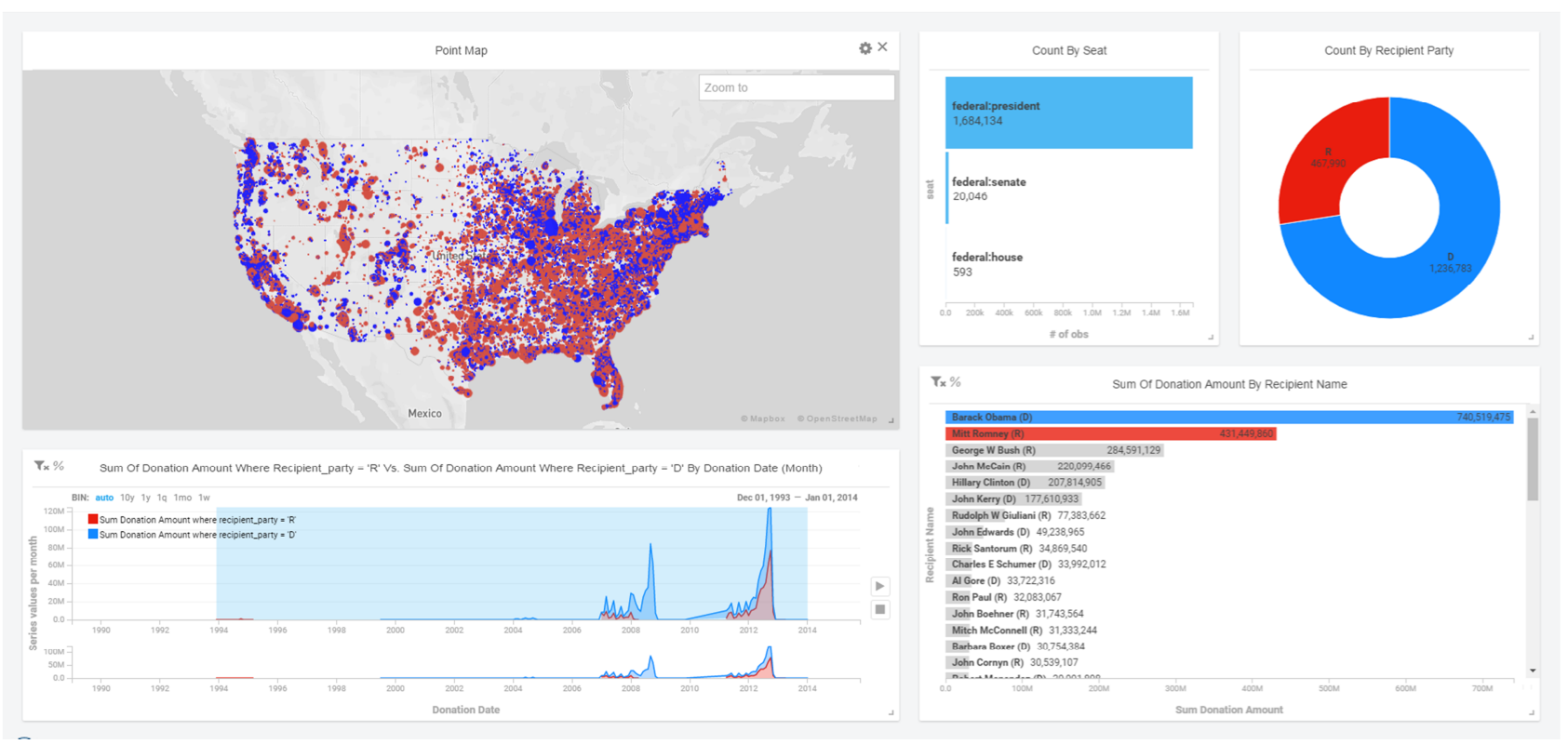

\section{Evaluation of InfoVis Tools from a Mental Models Perspective}

Whether an information visualization system supports or hinders the construction of a mental model depends on many different factors. In addition to the design, the complexity of the dataset (its dimensionality and size) and user characteristics (prior knowledge, tasks or goals, visual literacy) also play a decisive role. To figure out if and how an information visualization system supports the coherent construction of mental models, we recommend two different approaches. A heuristic evaluation by usability experts and/or a user study.

\subsection{Heuristic Evaluation}

A fast and forward method is an analytical evaluation of an information visualization tool by usability researchers based on a set of heuristics. There are different sets of heuristics available for information visualizations (see Forsell \& Johansson, 2010 for an overview and rating of the 10 most important heuristics). They include coherence-relevant heuristics (like consistency of design and minimal workload), but none explicitly focuses on the question, how an information visualization system supports the construction of mental models in a coherent way. Therefore, we suggest to extend existing heuristics with an evaluation of the presented coherence design elements. 


\subsection{User Studies}

Though a heuristic evaluation can provide first insights on how an information visualization system supports or hinders users' construction of mental models, its actual supportive function can only be assessed in real use. Different methods can assist our understanding how a user constructs a mental model (see Mayr, Schreder, Smuc \& Windhager, 2016 for an extended discussion of these methodologies): A mental model's structure and coherence can be best understood with sorting or sketching procedures, while its construction can be best understood with methods focusing on the process of mental model formation like observations or think aloud techniques.

Sorting or sketching techniques provide us with knowledge on the status quo of a mental model, that is, the outcome of interacting with an information visualization. Here, users are asked to sketch their mental model or to structure information elements to resemble their mental model. Some of these methodologies rely on a predefined list of elements (e.g., sorting techniques, inferential flow technique, or pairwise comparison); in others, users themselves generate a list of elements (e.g., repertory grid technique, concept maps). The relations between these elements provide insight into the mental models' coherence. By comparing users' mental models before and after interaction with an information visualization, it can be evaluated how the mental model was influenced by the system. This method can be complemented by interviews, where evaluators can inquire more deeply on the elements, their relations, and how the information visualization affected their construction.

If the actual construction of the mental models is of interest for the evaluation, sorting or sketching techniques should be replaced with or complemented by process methods. The user's interaction with the information visualization can be tracked via log files, screen recording, or eye tracking. For example, eye movements are not only indicators of visual attention, but also of cognitive load (Smuc, 2016). High cognitive load occurs in situations of incoherence between internal and external representations; when a user resolves this incoherence, a drop in cognitive load can be observed. In addition, users can be asked to think aloud while they interact with the system. We often let users freely explore an information visualization system and think aloud at the beginning of their interaction (Smuc et al., 2008). By this procedure, we are able to analyze the initial construction of mental models in the think-aloud protocols. This knowledge is especially valuable in early design phases, as affordances of the InfoVis system without further explanation are revealed. Inferences and mismatches in the think-aloud protocols are indicators, where an information visualization system supports coherence or is perceived incoherent and impedes construction. We developed a relational insight organizer (RIO, Smuc et al., 2009) to better understand how users of information visualizations connect their prior knowledge and insights to build up a global mental model.

Though this evaluation procedure might sound complex and time-consuming, we want to encourage designers to give it a try: They will learn a lot by observing only two or three users already. If no formal evaluation is needed, such a procedure can be carried out with little preparation and effort, but it will definitely pay off. 


\section{Discussion}

In this paper, we introduce the theory of mental models and elaborated on its potential for supporting visual communication in the domain of political data. The challenge to design accessible and coherent InfoVis systems grows with the increasing complexity of political topics and their underlying multidimensional datasets. If understanding a topic equals the successful construction of a mental model in terms of a locally and globally consistent representation, the designers of information visualizations should employ various consistency and coherence techniques as a sort of meta-design methods. Implemented in a reflected way, these techniques work like an infrastructure of cohesive forces between different views, supporting the cognitive functions of users to merge multiple perspectives and model components into a consistent bigger picture. While not aiming at an exhaustive collection of techniques or design recommendations, we discussed methods that show possible effects on the users' generation of more coherent mental models. While a large body of research exists in the field of discourse and text comprehension, a similar approach for InfoVis has been missing up to now. We focused on global coherence, which we define as the conceptual interrelation between multiple views, as it is most relevant for the user's interaction with multidimensional data.

Coherence is a crucial factor for the generation of complex mental models, but its application to the design of external representation has to be adjusted to the specifics of users and tasks: In political communication, the users are very heterogeneous, as are their goals. Some just want to inform themselves cursorily, others want to gain a thorough understanding and dive deeply into the ocean of data. Strong coherence support (e.g., the use of seamless transitions) can slow down the exploration and other forms of support (e.g., authored systems) reduce the users' freedom of action. Therefore, for political communication purposes, we would suggest to provide strong support of coherence at the beginning of the interaction with an InfoVis system (e.g., by an advance organizer), and to subsequently reduce the support to allow for more free exploration. Secondly, the required support depends on the user's expertise with diagrams, but also with the topic: Experts draw more inferences from less coherent information systems, whereas non-experts require more coherent systems to even build up a mental model (McNamara et al., 1996). Therefore, support should either be adapted to the user group (resulting in multiple different representations for each user group) or users should be able to select the amount of support they want and need. The question of supporting coherent representations should also be weighed against the users' expected tasks: For many simple tasks, cognitive collages may be an adequate internal representation and a simple diagram may be an adequate external representation. But when more global inferences and the generation of new multidimensional insights are involved, more coherent mental models are needed and more accessible and coherent InfoVis systems with multiple views seem adequate. For example, if a political information visualization system aims to support citizens in their opinion formation, those users who generated only cognitive collages could be more easily influenced by new arguments; whereas citizens who constructed a coherent mental model hold more elaborate and stronger opinions and are less likely to be influenced by cheap rhetoric. In this day and age - where Oxford dictionaries named "post-truth" the word of the year 2016 (Oxford Dictionaries Word of the Year, 2016) - generating a robust and coherent mental model might also become more and more important and a desirable goal for politically mature 
citizens. We are aware that political communication by cleverly thought out visualizations will hardly be the magic bullet against improper simplification and bending, messaging, and "enriching" the truth. But providing an elaborate and stable mental structure via visualizations to better reflect, classify and integrate the information flood could be an advantageous step in the right direction.

To investigate how external representations influence the generation of coherent mental models on a global level, the development of novel evaluation techniques is required. Existing methods like insights or task-oriented procedures are restricted to analyses of local coherence only. For an analysis of global coherence, we suggested two approaches: heuristic evaluations and user studies. In previous studies, we found it promising to let users think-aloud during their interaction with the InfoVis system and analyze the found propositions and relations (Smuc et al., 2009). The uttered inferences between their prior knowledge, insights, and their understanding of the tool allows further analyses of how users construct their mental models. By adding observations (like screen recordings), we can understand which design features support and which hinder the construction of mental models. If the core assumptions of our framework are supported by future empirical evidence, we plan to develop specialized heuristics for the evaluation of visualization tools.

Finally, we want to emphasize that - similar to political positions -- mental models are subjective representations of reality and are often not free of errors. It is an interesting question for further research whether incoherent external representations might even foster the generation of incoherent or insufficient mental models. Also, it is an open challenge to identify InfoVis interfaces that can lead users to question their existing mental models, identify mismatches, and correct existing misconceptions. Such an endeavor is of special importance in political communication, where these methods can help citizens to continuously question political information and their own opinions and act as informed and responsible citizens.

Similar to other media and methods, political information visualizations can also be potentially misused for the distorted representation of facts, even going far beyond the well-known "lie factor" (Tufte, 1991, p. 57f.), which was introduced to quantify distortion of data effects in diagrams. Drawing a coherent picture of invalid or deliberately distorted data and telling a convincing story, might lead citizens to view political spin or even blatant propaganda less critically. Although InfoVis aims for the amplification of cognition and communication in terms of a better, more detailed, and more objective comprehension of the underlying data, it could also be used to the contrary: to obfuscate facts and to distort and mislead its recipients. Nevertheless, this should not be used as an argument against a more coherent design of InfoVis in political communication, but as an additional motivation for the development of further standards of data and design transparency.

Against this background, we need not only well-established guidelines, curricula, and discourses how to not lie with data (Rogowitz, Treinish, \& Bryson, 1996), or even defense methods against "malviz" (i.e., malicious visualizations) due to InfoVis system attacks (Conti, Ahamad \& Stasko, 2005). But especially in the political communication realm we also need strict standards of good practice, to ensure transparency, traceability, and verifiability in terms of "correspondence 
with the facts" (cf. coherence- and correspondence-driven work domains and interface design, Vicente, 1990). This strategy is also emphasized in the critical InfoVis framework (Dörk, Feng, Collins, \& Carpendale, 2013), where the politics of visualization are explored and where four aspects are highlighted for InfoVis endeavors: disclosure, plurality, contingency, and empowerment. When designers of information visualizations for political communication are open about their data, aims and decisions, when they allow to view multiple perspectives of the data, so that citizens can explore the system from their own point of view, coherently designed InfoVis empower citizens to inform themselves, build an elaborated, well-founded mental model, and act accordingly.

\section{References}

Archambault, D., \& Purchase, H. C. (2012). Mental map preservation helps user orientation in dynamic graphs. In: W. Didimo and M. Patrignani (Eds.) International Symposium on Graph Drawing (pp. 475486). Berlin Heidelberg: Springer.

Ausubel, D. P. (1960). The use of advance organizers in the learning and retention of meaningful verbal material. Journal of Educational Psychology, 51, 267-272.

Bezdan, E., Kester, L., \& Kirschner, P. A. (2013). The influence of node sequence and extraneous load induced by graphical overviews on hypertext learning. Computers in Human Behavior, 29, 870-880.

Card, S. (2008). Information visualization. In A. Sears \& J.A. Jacko (Eds.), The human-computer interaction handbook: Fundamentals, evolving technologies, and emerging applications (pp. 509-544). New York: Lawrence Erlbaum.

Card, S., Mackinlay, J., \& Shneiderman, B. (1999). Readings in information visualization: Using vision to think. San Francisco: Morgan Kaufmann.

Coffey, J. W., Hoffman, R., \& Cañas, A. (2006). Concept map-based knowledge modeling: perspectives from information and knowledge visualization. Information Visualization, 5(3), 192-201.

Conti, G., Ahamad, M., \& Stasko, J. (2005, July). Attacking information visualization system usability overloading and deceiving the human. InProceedings of the 2005 symposium on Usable privacy and security (pp. 89-100). ACM.

Dörk, M., Feng, P., Collins, C., \& Carpendale, S. (2013, April). Critical InfoVis: exploring the politics of visualization. In CHI'13 Extended Abstracts on Human Factors in Computing Systems (pp. 2189-2198). ACM.

Forsell, C., \& Johansson, J. (2010). An heuristic set for evaluation in information visualization. In Proceedings of the International Conference on Advanced Visual Interfaces (pp. 199-206). ACM.

Gurlitt, J., Dummel, S., Schuster, S., \& Nückles, M. (2012). Differently structured advance organizers lead to different initial schemata and learning outcomes. Instructional Science, 40, 351-369.

Halloran, N. (2015). The Fallen of WWII, Available: http:/ /www.fallen.io/ww2/

Hegarty, M. (2011). The cognitive science of visual spatial displays: Implications for design. Topics in Cognitive Science, 3, 446-474. 
Johnson Laird, P. N. (1980). Mental models in cognitive science. Cognitive science, 4(1), 71-115.

Jones, N. A., H. Ross, T. Lynam, P. Perez, and A. Leitch. 2011. Mental models: an interdisciplinary synthesis of theory and methods. Ecology \& Society, 16(1), 46. [online] URL: http://www.ecologyandsociety.org/vol16/iss1/art46/

Kosara, R., \& Mackinlay, J. (2013). Storytelling: The next step for visualization. Computer, 46(5), 44-50.

Liu, Z., \& Stasko, J. T. (2010). Mental models, visual reasoning and interaction in information visualization: A top-down perspective. IEEE Transactions on Visualization \& Computer Graphics, 16, 999-1008.

Magliano, J. P., Miller, J., \& Zwaan, R. A. (2001). Indexing space and time in film understanding. Applied Cognitive Psychology, 15(5), 533-545.

Mayr, E., Schreder, G., Smuc, M., Windhager, F. (2016). Looking at the representations in our mind: Measuring mental models of information visualizations. In M. Sedlmair, P. Isenberg, T. Isenberg, N. Mahyar, \& H. Lam (Eds.), Proceedings of the Beyond Time and Errors on Novel Evaluation Methods for Visualization (pp. 96-103). New York: ACM.

McManus, T. F. (2000). Individualizing instruction in a web-based hypermedia learning environment: Nonlinearity, advance organizers, and self-regulated learners. Journal of Interactive Learning Research, $11,219-251$.

McNamara, D. S., Kintsch, E., Songer, N. B., \& Kintsch, W. (1996). Are good texts always better? Interactions of text coherence, background knowledge, and levels of understanding in learning from text. Cognition \& instruction, 14(1), 1-43.

North, C., \& Shneiderman, B. (2000). Snap-together visualization: can users construct and operate coordinated visualizations?. International Journal of Human-Computer Studies, 53(5), 715-739.

Oxford Dictionaries Word of the Year 2016 is post-truth. (2016, November 16). Retrieved from https://www.oxforddictionaries.com/press/news/2016/11/17/WOTY-16

Pohl, M., Smuc, M., \& Mayr, E. (2012). The user puzzle. Explaining the interaction with visual analytics systems. IEEE Transactions on Visualization \& Computer Graphics, 18, 2908-2916. doi: 10.1109/TVCG.2012.273

Rogowitz, B. E., Treinish, L. A., \& Bryson, S. (1996). How not to lie with visualization. Computers in Physics, 10(3), 268-273.

Ruchikachorn, P., \& Mueller, K. (2015). Learning visualizations by analogy: Promoting visual literacy through visualization morphing. IEEE transactions on visualization and computer graphics, 21(9), 10281044.

Segel, E., \& Heer, J. (2010). Narrative visualization: Telling stories with data. IEEE Transactions on Visualization \& Computer Graphics, 16, 1139-1148.

Shneiderman, B. (1996). The Eyes Have It: A Task by Data Type Taxonomy for Information Visualizations. In Proceedings of the IEEE Symposium on Visual Languages (pp.336-343). Washington: IEEE Computer Society Press. 
Smuc, M. (2016). Just the other side of the coin? From error to insight analysis. Information Visualization, 15, 312-324. DOI: 1473871615598641.

Smuc, M., Mayr, E., Lammarsch, T., Aigner, W., Miksch, S., \& Gärtner, J. (2009). To score or not to score? Tripling insights for participatory design. IEEE Computer Graphics \& Application, 29(3), 29-38. doi: 10.1109/MCG.2009.53

Smuc, M., Mayr, E., Lammarsch, T., Bertone, A., Aigner, W., Risku, H., \& Miksch, S. (2008). Visualizations at first sight: Do insights require training? In Proceedings of USAB (pp. 261-280). Berlin: Springer.

Spence, R. (1999). A framework for navigation. International Journal of Human-Computer Studies, 51, 919945.

Tufte, E. (1991). The Visual Display of Quantitative Information, 2nd Edition, Graphics Press, USA.

Tversky, B. (1993). Cognitive maps, cognitive collages, and spatial mental models. In A. U. Frank \& I. Campari (Eds.), Spatial information theory. A theoretical basis for GIS (pp. 14-24). Berlin: Springer.

Tversky, B. (2011). Visualizing thought. Topics in Cognitive Science, 3, 499-535.

Ubaldi, B. (2013). Open Government Data: Towards Empirical Analysis of Open Government Data Initiatives. OECD Working Papers on Public Governance, No. 22, OECD Publishing. http://dx.doi.org/10.1787/5k46bj4f03s7-en

Urakami, J., \& Krems, J. F. (2012). How hypertext reading sequences affect understanding of causal and temporal relations in story comprehension. Instructional Science, 40, 277-295.

van Dijk,T. A., \& Kintsch, W. (1983). Strategies of discourse comprehension. New York: Academic Press.

Van Merriënboer, J. J., \& Kirschner, P. A. (2012). Ten steps to complex learning: A systematic approach to four-component instructional design. New York: Routledge.

Vicente, K. J. (1990). Coherence-and correspondence-driven work domains: Implications for systems design. Behaviour \& Information Technology, 9(6), 493-502.

Windhager, F., \& Mayr, E. (2012). Cultural heritage cube. A conceptual framework for visual exhibition exploration. In 2012 16th International Conference on Information Visualisation (pp. 540-545). IEEE.

Windhager, F., \& Smuc, M. (2014). The arts of the possible. Information visualization in the field of politics. JeDEM - eJournal of eDemocracy and Open Government, 6, 151-165.

Zwaan, R. A. (1999). Five dimensions of narrative comprehension: The event-indexing model. In S. R. Goldman, A. C. Graesser \& P. van den Broek, (Eds.), Narrative comprehension, causality, and coherence: Essays in honor of Tom Trabasso (pp. 93-110). London: Lawrence Erlbaum Associates.

Zwaan, R. A., \& Radvansky, G. A. (1998). Situation models in language comprehension and memory. Psychological Bulletin, 123, 162-185. 


\begin{abstract}
About the Authors
Günther Schreder

Günther Schreder works as a research associate at the Center for Cognition, Information and Management, Danube University Krems, Austria. He received his diploma in psychology at the University of Vienna, Austria. His research and teaching activities focus on user-centered design with respect to non-expert users, narrative information design and the development and investigation of cognitive models of narrative communication and organizational culture.
\end{abstract}

\title{
Florian Windhager
}

Florian Windhager works as a research associate at the Department for Knowledge and Communication Management at Danube University Krems, Austria. He obtained his degree in Philosophy (M.A.) from the University of Vienna. His research interests include applications and cognitive foundations of information visualization, information visualization in the digital humanities, and concept development for visual analytics interfaces.

\section{Michael Smuc}

Michael Smuc graduated in Psychology at the University of Vienna with an emphasis on methodology, empirical research and cognitive science. He worked for several years as a traffic psychologist with the Austrian Road Safety Board, managing interdisciplinary projects addressing driver interaction with new technologies and Telematics. In 2007, he joined the Danube-University Krems, Austria as a research associate focusing on usability, human computer interaction and participatory information design.

\section{Eva Mayr}

Eva Mayr graduated in Psychology from the University of Vienna, Austria, in 2004 and completed her doctorate in Applied Cognitive and Media Psychology at the University of Tuebingen, Germany, in 2009. From 2002 to 2005, she worked at the Institute of Psychology in Vienna. Since 2008, she has been a research associate in the Department of Knowledge and Communication Management at the Danube University Krems, Austria. Her research interests focus on how new media technologies can support cognitive processing and informal learning. 